\title{
COVID- 19 and the abnormalities of some biochemical tests: review article
}

\author{
Alaa Shawqi Abdulbari \\ Department of Pharmacy \\ Al-Esraa University collage \\ Baghdad, Iraq \\ alaa.shawqi@esraa.edu.iq
}

\author{
Noor N.O. Al-Saadi \\ Department of Medical Lab. \\ Technologies \\ Bilad Al-Rafidain University College \\ Diyala, Iraq \\ noor.naief@hotmail.com
}

\begin{abstract}
Coronaviruses are a group of viruses proven to affect both respiratory and gastrointestinal diseases in a various animal and human organisms. More than 100 million people worldwide are currently believed to have been infected and more than two million people have died and induced clinical syndrome of coronavirus disease in 2019.It's (COVID19). The purpose of this analysis was to determine whether multiple biochemical test derangements are a common feature in patients with reported COVID-19 infection, to determine the relationship between the deranged liver test and lipoprotein with severity of COVID-19 outcome, and to determine whether liver failure or irregular lipids levels is a common feature of COVID-19. This review was conducted using the Web of Science, PubMed and Scopus databases. English case-series and cross-sectional papers were considered, describing the currently offered findings on the relationship between certain biomedical tests and COVID-19 infections. In summary, COVID-19 may have a severe tendency in elderly patients with biochemical indices (decrease albumin, decrease LDL-c, HDLc, and TC, elevated levels of CRP, AST, LDH and CK). These parameters could use as indicators to predict the severity of the disease.
\end{abstract}

\section{Keywords-COVID-19, Liver function, lipid profile.}

\section{Introduction:}

Coronaviruses are a group of viruses proven to affect both respiratory and gastrointestinal diseases in varied animal and human organisms [1]. These viruses appear to strike the upper respiratory tract causing pneumonia anywhere from slight to serious diseases such as cold or pneumonia in more extreme cases. The World Health Organization (WHO) identifies pneumonia as coronavirus disease 2019 (COVID-19) [2]. Such cases have been recorded in many countries since the outbreak of epidemic. Clinical manifestations and severity of COVID-19 are close to that of SARS-CoV [3]. China has reported a variety of factors that are associated with the severity of COVID-19. Risk factors for severe COVID-19 show to be male sex, older age, low saturation of oxygen, and the abnormal laboratory findings [4].

\section{EFFECT OF COVID-19 ON LIPID PROFILE:}

Lipid plays a key role in viral infection. Altered levels of serum lipids, in particular, cholesterol levels, indeed, human immunodeficiency virus (HIV) and hepatitis C virus (HCV)infections have been estimated to differ. Some proteins have been suggested to be involved in $\mathrm{HCV}$ infection, major high-density lipoprotein (HDL) receptor, such as SR-BI [5]. Cholesterol has also been shown to be a critical component of pathogenic viruses that attack host cells [6].

In a 2017 study $\mathrm{Wu}$ et al. 2017 [7], serum metabolic analyzes were conducted in 25 recovered SARA patients 12 years after infection. Their data showed that levels of very low-density lipoprotein (VLDL) cholesterol and triglyceride (TG) were significantly higher than those of healthy controls. Similarly, some studies have suggested that antiviral therapy in HIV-infected patients has induced significant abnormal lipid levels [8], A serum lipid profile in HCV patients as well as abnormal [9], which may contribute to cardiovascular disease in patients.

A retrospective study was performed by Xiuqi et al 2020 on 597 patients with COVID-19, In COVID-19 patients, HDL-c, LDL-c and TC levels were shown to be significantly lower than in normal subjects, and HDL-c levels significantly decreased in critical cases relative to moderate and severe patients, While Triglyceride has been shown to be significantly lower in normal subjects. Data indicate that reductions in lipid levels are correlated with the severity of symptoms in patients. with COVID-19, so that they speculate that one potential mechanism underpinning research data is that more extreme inflammation and the worse vascular permeability, more plasma cholesterol and lipids leak into alveolar space, during disease progression and less LDL-c and cholesterol remain in the plasma during the progression of the disease. Irregular lipid levels may play a significant role in the pathological progression of COVID-19, a process that urgently needs to be investigated, they argue [10].

In a clinical retrospective study, A total of 228 adults with COVID-19 infection studied by Jia Teng et al, 2020 [20] compared to control, COVID-19 provided significantly lower levels of cholesterol, triglyceride, LDL-C, and HDLC. In this analysis, the levels of HDL-C in adult patients with COVID-19 were lower than average at admission, which was close to [10] results. 
Sébastien et al. 2020 [12] studied 48 COVID-19 patients as a prospective study, levels of lipoprotein were evaluated on admission and every day for 28 days at the ICU, and the study showed that lipoprotein concentrations were low upon entry, usually below reference range. A statistically significant increase during the ICU stay was observed in LDL-C and HDL-C over time. No correlation was found between concentrations of LDL-C, HDL-C and mortality on day 28, which was close to [13] results.

Generally, HDL is a lipoprotein that is anti-inflammatory and protects against oxidized lipids, and negatively controls the activation of $\mathrm{T}$ cells in macrophages and dendritic cells and the expression of inflammatory mediators [14, 15]. Whereas HDL may be oxidized during systemic inflammation, becomes dysfunctional oxidized HDL (oxHDL), including oxidized cholesterol [16]. It has been demonstrated that oxidized HDL and oxidized LDL increase immune activation [17].

This irregular lipid levels have many potential causes. First, COVID-19 can harm the function of the liver and thereby reduce the biosynthesis of LDL-c. Second, COVID19-induced acute inflammation affects lipid metabolism [8]. Thirdly, lipids are particularly susceptible to free radical degradation, the levels of which are typically elevated in viral infected host cells [18].

T. Chida, and X. Wei et al. [10, 19] found vascular permeability may be altered by COVID-19 infection, allowing cholesterol molecules to leak into tissues to form exudate, such as alveolar spaces.

\section{EFFECT OF COVID-19 ON LIVER FUNCTION:}

The COVID-19 receptor, angiotensin-converting enzyme 2 (ACE2), is considered to be a chief receptor of the viral spike protein and is important for infection [20, 21]. The ACE2 protein in the biliary system, colon and liver is present at high levels [22] and the RNA shedding is well known in the GI tract [23]. These data indicate that for the liver and GI tract, COVID-19 may have tropism and that might be locations of active viral replication and indirect or direct injury to tissue [24].

S. Wan et al. [20] mentioned that hepatic injury is detected from the time of initial medical system contact due to infection with COVID-19, this suggests that the initial insult is not linked to medical managers, but is owing to either the direct influence of the infection or systemic illness. However many, other factors potentially affect the course of liver injury. There is a direct viral cytopathic influence, given the known existence of the ACE2 receptor in the liver [20, 25]. Viral RNA has been found in liver tissue in SARS infection [26]. Moreover, recent data indicates that mitochondrial proteins can directly act together with the virus [27]. Provide the AST-dominant injury profile with a possible mechanistic interpretation. Instead, a key role can be played by the strong inflammatory response found in COVID-19. A very high level of IL-66 characterizes the immune response to COVID-19 (which has been involved in both remedial liver disease and inflammatory responses) [24].

The presence of liver damage has been confirmed in extreme COVID-19 cases in recent studies, often with higher aspartate aminotransferase (AST), alanine aminotransferase (ALT), LDH, D-dimer, C-reactive protein (CRP) and lower albumin and total bilirubin levels [1, 28, 29]. In addition, it was reported that the severity of COVID-19 was associated with higher levels of AST, CRP and D-dimer.

W. Guan et al. [30] examined 1099 COVID-19 patients where most patients had elevated CRP levels; elevated ALT, AST, creatine kinase $(\mathrm{CK})$, and D-dimer levels were less common.

Jong et al. [4] analyzed 110 COVID-19 patients were split into two categories: severe and non-severe, this resulted in a significantly lower albumin level in the severe group than in the non-severe group. AST, blood urea nitrogen, total bilirubin, lactate dehydrogenase (LDH), and creatine kinase$\mathrm{MB}$ (CK-MB) concentrations in the non-severe group were significantly lower than in the severe group. With respect to $\mathrm{C}$-reactive protein infection-related markers, the amount was significantly higher in the severe group than in the nonsevere group.

Zheng et al. [3] analyzed 161 COVID-19 patients with COVID-19 and grouped patients into two groups: severe and non-severe, elevated levels of CRP (75.2\%) and LDH (23.6\%), and decreased lymphocyte count (26.1 percent). AST, CK-MB and LDH were increased in comparison to the non-severe C-reactive protein group, with substantial differences $(\mathrm{p}<0.05)$.

L. Xiaochen Li et al, [31] examined 548 COVID-19 patients that classified into two groups, the non-severe and the severe group. All patients had elevated levels of LDH, globulin, NT-pro B-type natriuretic peptide, D-dimer, ALT, AST, total bilirubin, blood urea nitrogen, conjugate bilirubin and creatinine.

Y. Wei et al. [2] analyzed 276 COVID-19 patients, and classified patients into 2 groups, severe and non-severe. In 60.9 percent $(162 / 266)$ of patients, CRP levels were increased. The elevated level of CK, ALT, AST and myoglobin have been observed.

W. Yaping et al.[32] studied 275 COVID-19 patients with blood chemistry (LDH, CK, AST, Albumin, ALT) and $\mathrm{C}$-reactive protein within normal ranges. However, the admission levels of LDH, CK, and aspartate aminotransferase (AST) in the severe group were higher than in the non-severe group. In $51.3 \%$ of all patients, albumin (ALB) was reduced and more patients had reduced albumin levels in the severe group than those in the non-severe group.

Gatta et al., 2012 [33] it was confirmed that one of the most substantial ones significant features influencing the diagnosis of sepsis patients was hypoalbuminemia. J.S.M et al.[34] found that a drop in the level of albumin (ALB) in sepsis patients suggested a worsening and poor prognosis of the disease. The sepsis-related mechanism of ALB reduction is considered to be due to the body's excessive inflammatory response. The inflammatory reaction contributes to the release from the cells of Kupffer of oxygen-free radicals and prostaglandins, which can prevent the liver synthesis of ALB and encourage protein intake and decomposition, leading to hypoalbuminemia. Therefore, dynamically assessing ALB levels in these patients is necessary.

There were elevated levels of LDH, ALT, AST, total bilirubin, conjugated bilirubin, creatinine and blood urea nitrogen. In various diseases, including cancer and infections, LDH has been recognized as a marker of the serious prognosis [35]. In severe cases with COVID-19, the 
increased level of LDH suggested that LDH may be associated with the lung injury and tissue damage, ensuring that the potential mechanism to be investigated.

In another hypothesis, Cytotoxic $\mathrm{T}$ cells caused by viruses and the imbalanced innate immune system are postulated to lead to collateral liver damage, indicating the correlation of liver marker defects with the seriousness of COVID-19 disease.

Several medications used in patients with COVID-19 may also have liver damage, Including lopinavir/ritonavir, reportedly leading to liver damage and influence liver testing, associated with seven times greater odds of liver injury $[1,36]$.

Centered on previous ACE2 receptor studies, small amounts of these receptors have indeed been confirmed to be expressed in human hepatocytes [37], suggesting the nonsignificant effect of COVID-19 infection on liver function in non-severe and mild cases [38].

Some studies have reported that in COVID-19 patients who suffer pneumonia, LDH was higher. $\mathrm{LDH}$ is an important component of the metabolism of glucose and is widely expressed in the tissues of the organ, particularly in the liver and myocardial cells. The cytoplasmic membrane damage could release LDH within cells [40]. Elevated levels of LDH were also identified on the basis of earlier studies on MERS and SARS. It can therefore be inferred that LDH might function independently as with weak clinical outcomes as a risk factor, and raised the alarm for more study [38]. the widespread expression in ACE2 receptors in cardiac blood vessels may cause higher LDH levels [40]. In another hypothesis, myositis that resulted from infection with the virus was responsible for the increased amount of LDH [41]. In addition, its concentrations will be increased due to the coexistence of ACE2 receptors in hepatocytes as a result of hepatocyte injury. It also relieves the fact that liver and heart damage will occur as a direct effect of COVID-19 on targeted organ damage [39]. In severe cases, therefore, elevated LDH enzymes could occur as a result of guided liver or extrahepatic damage.

\section{Conclusions:}

To summarize, COVID-19 may have a severe tendency in the elderly patient biochemical indexes (decrease albumin, decrease LDL-c, HDL-c, and TC, increased CRP, increased AST, increased LDH and CK) could be used as indicators to predict the severity of the disease.

\section{REFERENCES}

[1] Q. Cai, D. Huang, H. Yu, Z. Zhu, Z. Xia, Y. Su, Z. Li, et al., "COVID-19: Abnormal liver function tests". J Hepatol, vol. 73(3), pp. 566-574, 2020.

[2] Y. Wei, W. Zeng, X. Huang, J. Li, X. Qiu, H. Li, et al., "Clinical characteristics of 276 hospitalized patients with coronavirus disease 2019 in Zengdu District, Hubei Province: a single-center descriptive study". BMC Infect Dis, vol. 20(1), pp. 549, 2020.

[3] F, Zheng, W Tang, H Li, Y-X Huang, Y-L Xie and Z-G Zhou, "Clinical characteristics of 161 cases of corona virus disease 2019 (COVID-19) in Changsha”. Eur Rev Med Pharmacol Sci, vol. 24(6), pp. 3404-3410, 2020.

[4] J.G. Jang, J. Hur, E.Y. Choi, K.S. Hong, W. Lee and J.H. Ahn, "Prognostic Factors for Severe Coronavirus Disease 2019 in Daegu, Korea”. J Korean Med Sci, vol. 35(23), pp. e209, 2020.

[5] M. Dreux, V.L. Dao, J. Fresquet, M. Guérin, Z. Julia, G. Verney, et al. "Receptor complementation and mutagenesis reveal SR-BI as an essential HCV entry factor and functionally imply its intra- and extracellular domains”. PLoS Pathog, vol. 5(2), pp. e1000310, 2009.

[6] G. Meher, S. Bhattacharjya and H. Chakraborty. "Membrane Cholesterol Modulates Oligomeric Status and Peptide-Membrane Interaction of Severe Acute Respiratory Syndrome Coronavirus". Fusion Peptide, J Phys Chem B, vol. 123(50), pp.10654-62, 2019.

[7] Q. Wu, L. Zhou, X. Sun, Z. Yan, C. Hu, J. Wu, et al. "Altered Lipid Metabolism in Recovered SARS Patients Twelve Years after Infection". Sci Rep, vol. 7(1), pp. 9110, 2017.

[8] N.T. Funderburg and N.N. Mehta "Lipid Abnormalities and Inflammation in HIV Inflection". Curr HIV/AIDS Rep, pp. 13(4), pp. 218-25, 2016

[9] T. Chida, K. Kawata, K. Ohta, E. Matsunaga, J. Ito, S. Shimoyama, et al., "Rapid Changes in Serum Lipid Profiles during Combination Therapy with Daclatasvir and Asunaprevir in Patients Infected with Hepatitis C Virus Genotype 1b". Gut Liver, vol. 12(2), pp. 201-7, 2018.

[10] X. Wei, W. Zeng, J. Su, H. Wan, X. Yu, X. Cao, et al., "Hypolipidemia is associated with the severity of COVID-19". J Clin Lipidol, vol. 14(3), pp. 297-304, 2020.

[11] J. Sun, Z. Chen, P. Nie, H. Ge, L. Shen, F. Yan, et al., "Lipid Profile Features and Their Associations With Disease Severity and Mortality in Patients With COVID-19". Frontiers in Cardiovascular Medicine, vol. 7, 584987, 2020.

[12] T. Sébastien, C. De Tymowski, M. Assadi, N. Zappella, S. JeanBaptiste, T. Robert, et al., "Lipoprotein concentrations over time in the intensive care unit COVID-19 patients: Results from the ApoCOVID study". PLoS One, vol. 15(9), pp. e0239573, 2020.

[13] N.A. Taborda, Y. Blanquiceth, S. Urcuqui-Inchima, E. Latz, J.C. Hernandez, High-Density Lipoproteins Decrease Proinflammatory Activity and Modulate the Innate Immune Response, J. Interferon Cytokine Res. 39(12) (2019) 760-70.

[14] S.G. Thacker, A. Zarzour, Y. Chen, M.S. Alcicek, L.A. Freeman, D.O. Sviridov, et al., "High-density lipoprotein reduces inflammation from cholesterol crystals by inhibiting inflammasome activation". Immunology, vol. 149(3), pp. 306-19, 2016.

[15] T. Kelesidis, J.S. Currier, D. Huynh, D. Meriwether, C. CharlesSchoeman, S.T. Reddy, et al., "A biochemical fluorometric method for assessing the oxidative properties of HDL". J Lipid Res, vol. 52(12), pp. 2341-51, 2011.

[16] T. Kelesidis, N. Jackson, G.A. McComsey, X. Wang, D. Elashoff, M.P. Dube, et al., "Oxidized lipoproteins are associated with markers of inflammation and immune activation in HIV-1 infection". AIDS, vol. 30(17), pp. 2625-2633, 2016.

[17] D.A. Zidar, S. Juchnowski, B. Ferrari, B. Clagett, H.A. Pilch-Cooper, S. Rose, et al. "Oxidized LDL levels are increased in HIV infection and may drive monocyte activation". J Acquir Immune Defic Syndr. Vol. 69, pp. 154-160. 2015.

[18] S. Tian, W. Hu, L. Niu, H. Liu, H. Xu and X. Shu-Yuan, "Pulmonary pathology of early phase 2019 novel coronavirus (COVID-19) pneumonia in two patients with lung cancer". J Thorac Oncol, vol. 15, pp. 700-704, 2020.

[19] R. Graham, F.E. Donaldson and R.S. Baric, "A decade after SARS: strategies for controlling emerging coronaviruses". Nat Rev Microbiol, vol. 11 pp. 836-848, 2013.

[20] S. Wan, Y. Xiang, W. Fang, Y. Zheng, B. Li, Y. Hu, et al., "Clinical features and treatment of COVID-19 patients in northeast Chongqing”. J Med Virol, vol 92(7), pp. 797-806, 2020.

[21] C. Qin, H. Minghan, Z. Ziwen, L. Yukun, “Alteration of lipid profile and value of lipids in the prediction of the length of hospital stay in COVID-19 pneumonia patients". Food Sci. Nutr, vol. 8, pp. 61446152,2020

[22] H. Xu, L. Zhong, J. Deng, J. Peng, H. Dan, X. Zeng, et al., "High expression of ACE2 receptor of 2019-nCoV on the epithelial cells of oral mucosa". Int J Oral Sci, vol. 12, pp. 8, 2020.

[23] F. Xiao, M. Tang, X. Zheng, Y. Liu, X. Li and H. Shan, "Evidence for gastrointestinal infection of COVID-19". Gastroenterology, vol. 158, pp. 1831-1833, 2020.

[24] A.K. Schaefer, A. Arvind, P.P. Bloom and R.T. Chung, "Interrelationship between Coronavirus Infection and Liver Disease". Clinical Liver Disease, vol. 15(5), 2020. 
[25] J. Gu, B. Han and J. Wang J, "COVID-19: gastrointestinal manifestations and potential fecal-oral transmission" Gastroenterology, vol. 158, pp. 1518-1519, 2020.

[26] C. Tai-Nin, L. Kam-Cheong, H. Yao, T. Tak-Yin, C. Tat-Chong, Y. Yiu-Cheong, et al., "SARS-associated viral hepatitis caused by a novel coronavirus: report of three cases". Hepatology, vol. 39, pp. 302-310, 2004.

[27] D.E. Gordon, G.M. Jang, M. Bouhaddou, J.Xu, K. Obernier, K.M White, et al., "A SARS-CoV-2 protein interaction map reveals targets for drug repurposing". Nature, vol. 583(7816), pp. 459-468, 2020.

[28] Y.Zhang, L. Zheng, L. Li, M. Zhao, J. Xiao and Q.Zhao, "Liver impairment in COVID-19 patients: A retrospective analysis of 115 cases from a single centre in Wuhan city, China". Liver Int., vol. 40(9), pp. 2095-2103, 2020.

[29] Y. Feng, Y. Ling, T. Bai, Y. Xie, J. Huang, J. Li, et al., "COVID-19 with Different Severities: A Multicenter Study of Clinical Features". Am J Respir Crit Care Med., vol. 201(11), pp. 1380-1388, 2020.

[30] H. Yue, X. Bai, J. Wang, Q. Yu, W. Liu, J. Pu, et al., "China Medical Treatment Expert Group for Covid-19. Clinical Characteristics of Coronavirus Disease 2019 in China". N Engl J Med, vol. 382, pp. 1708-1720, 2020.

[31] L. Xiaochen, S. Xu, M. Yu, K. Wang, Y. Tao, Y. Zhou, et al., "Risk factors for severity and mortality in adult COVID-19 inpatients in Wuhan”. J Allergy Clin Immunol, vol. 146(1), pp. 110-118, 2020.

[32] W. Yaping, B. Liao, Y.Guo, F. Li, C. Lei, F. Zhang, et al., "Clinical Characteristics of Patients Infected With the Novel 2019 Coronavirus (COVID-19) in Guangzhou, China". Open Forum Infect Dis, vol. 7(6), pp. ofaa187, 2020.

[33] A. Gatta, A. Verardo and M. Bolognesi, "Hypoalbuminemia". Intern Emerg Med, vol, 7(Suppl 3), pp. S193-9, 2012.
[34] J.S.M. Peiris, S.T. Lai, L.L.M. Poon, Y. Guan, L.Y.C. Yam, W. Lim, et al., "SARS study group. Coronavirus as a possible cause of severe acute respiratory syndrome". Lancet, vol. 361, pp. 1319-25, 2003.

[35] A. Erez, O. Shental, J.Z. Tchebiner, M. Laufer-Perl, A. Wasserman, T. Sella, et al., "Diagnostic and prognostic value of very high serum lactate dehydrogenase in admitted medical patients". Isr Med Assoc J. vol. 16, pp. 439-43, 2014.

[36] P. Meraviglia, M. Schiavini, A. Castagna, P. Vigano, T. Bini, S. Landonio, et al., "Lopinavir/ritonavir treatment in HIV antiretroviralexperienced patients: evaluation of risk factors for liver enzyme elevation”. HIV medicine, vol. 5(5), pp. 334-43, 2004.

[37] J.A. Grace, C.B. Herath, K.Y. Mak, L.M. Burrell and P.W. Angus, "Update on new aspects of the renin-angiotensin system in liver disease: clinical implications and new therapeutic options". Clinical science, vol. 123(4) pp. 225-39, 2012.

[38] Z. Yafei, L. Zheng, L. Li, M. Zhao, J. Xiao and Q. Zhao, "Liver impairment in COVID-19 patients: A retrospective analysis of 115 cases from a single centre in Wuhan city, China". Liver Int, vol. 40(9), pp. 2095-2103, 2020.

[39] Y. Han, H. Zhang, S. Mu, W. Wei, C. Jin, C. Tong, et al., "Lactate dehydrogenase, an independent risk factor of severe COVID-19 patients: a retrospective and observational study". Aging (Albany NY), vol. 12(12), pp. 11245-11258, 2020.

[40] I. Hamming, W. Timens, M. Bulthuis, A. Lely, G. Navis and H. Goor, "Tissue distribution of ACE2 protein, the functional receptor for SARS coronavirus. A first step in understanding SARS pathogenesis". J Pathol, vol. 203(2), pp. 631-7, 2004.

[41] M.N. Bangash, J. Patel and D. Parekh, "COVID-19 and the liver: little cause for concern". The Lancet Gastroenterol Hepatol, Vol. 5(6), pp. 529-530, 2020. 